\title{
The influence of care interventions on the continuity of sleep of intensive care unit patients ${ }^{1}$
}

\author{
Fernanda Luiza Hamze ${ }^{2}$ \\ Cristiane Chaves de Souza ${ }^{3}$ \\ Tânia Couto Machado Chianca ${ }^{4}$
}

Objective: to identify care interventions, performed by the health team, and their influence on the continuity of sleep of patients hospitalized in the Intensive Care Unit. Method: descriptive study with a sample of 12 patients. A filming technique was used for the data collection. The awakenings from sleep were measured using the actigraphy method. The analysis of the data was descriptive, processed using the Statistical Package for the Social Sciences software. Results: 529 care interventions were identified, grouped into 28 different types, of which 12 (42.8\%) caused awakening from sleep for the patients. A mean of 44.1 interventions/patient/day was observed, with 1.8 interventions/patient/hour. The administration of oral medicine and food were the interventions that caused higher frequencies of awakenings in the patients. Conclusion: it was identified that the health care interventions can harm the sleep of ICU patients. It is recommended that health professionals rethink the planning of interventions according to the individual demand of the patients, with the diversification of schedules and introduction of new practices to improve the quality of sleep of Intensive Care Unit patients.

Descriptors: Sleep; Nursing Care; Intensive Care Units.

\footnotetext{
1 Paper extracted from doctoral dissertation "Influência das intervenções assistenciais sobre a continuidade do sono de pacientes em centro de terapia intensiva", presented to Escola de Enfermagem, Universidade Federal de Minas Gerais, Belo Horizonte, MG, Brazil.

2 MSc, RN, Programa SOS Urgência, Ministério da Saúde, Brasília, DF, Brazil.

${ }^{3}$ Doctoral student, Escola de Enfermagem, Universidade Federal de Minas Gerais, Belo Horizonte, MG, Brazil. Assistant Professor, Universidade Federal de Viçosa, Viçosa, MG, Brazil. Scholarship holder from Coordenação de Aperfeiçoamento de Pessoal de Nível Superior (CAPES), Brazil. ${ }^{4}$ PhD, Full Professor, Escola de Enfermagem, Universidade Federal de Minas Gerais, Belo Horizonte, MG, Brazil.
}

Corresponding Author: Cristiane Chaves de Souza Universidade Federal de Viçosa Departamento de Medicina e Enfermagem Av. Peter Henry Rolfs, s/n Campus Universitário CEP: 36570-900, Viçosa, MG, Brasil E-mail: souzac.cris@gmail.com
Copyright @ 2015 Revista Latino-Americana de Enfermagem This is an Open Access article distributed under the terms of the Creative Commons Attribution Non-Commercial License (CC BY-NC).

This license lets others distribute, remix, tweak, and build upon your work non-commercially, and although their new works must also acknowledge you and be non-commercial, they don't have to license their derivative works on the same terms. 


\section{Introduction}

Intensive Care Units (ICU) are intended for the care of critically ill patients or those at risk of death. They offer uninterrupted medical and nursing care with specific equipment, specialized human resources and the use of technologies for diagnosis and therapy(1). Patients hospitalized in the ICU may present changes in different bodily systems, among which, those related to sleep stand out(2-4).

Sleep is essential for the rest, well-being, preservation of homeostasis and balance of the different bodily systems of the person, and is characterized as being a cyclical physiological brain activity, accompanied by behavioral manifestations $s^{(2,5-6)}$. The sleep stages compose a sleep cycle that lasts, on average, between 90 and 110 minutes and is repeated four to six times during the night. The restorative function of sleep is not only based on its ideal time, but also on its normal distribution through out all the stages ${ }^{(5-7)}$.

Among the methods that have been used in studies for the measurement of sleep polysomnography and actigraphy stand out ${ }^{(7-10)}$. Polysomnography has been considered the gold standard for identifying the stages of sleep and its disorders. This method continuously and simultaneously evaluates brain electrical activity, eye movement and muscle tone ${ }^{(7)}$. However, its use in studies developed in the ICU is costly, as the patient must remain monitored for 24 hours, with equipment, specific software, and specialized technical teams being necessary for the examination and the interpretation of the examination ${ }^{(8)}$. Actigraphy is a noninvasive method of monitoring the sleep/wake cycle. It uses the actigraph, a device placed on the wrist of the patient to detect body movements through means of an accelerometer system. It is an alternative method to polysomnography, due to its ease of use and ability to gather data over long periods of time. The limitations of the device include the lack of information about the stages and depth of the sleep ${ }^{(9-10)}$.

Sleep deprivation can cause physiological changes in the individual, such as abnormalities in the immune system, psychological and thermoregulation disorders, changes in metabolism and reduced quality of life $\mathrm{l}^{(4-6)}$. The intensive care environment has been regarded as disturbing for the sleep patterns of patients( ${ }^{(2,4,11)}$.

Among the various professionals that provide care for ICU patients, those of nursing stand out. The change in sleep is identified as a nursing problem, which implies the need to carry out interventions directed toward its improvement/resolution. The NANDA-International (NANDA-I) taxonomy of nursing diagnoses ${ }^{(12)}$ presents the diagnosis "disturbed sleep pattern", defined as "time-limited disruptions in the quantity and quality of sleep, due to external factors".

During clinical practice, sleep interruptions of patients are observed, both at night and during the day, due to the care or evaluations conducted by health professionals. It was found that patients are awakened from sleep to perform care interventions that could be delayed. Furthermore, some patients reported they did not rest at night, because they had trouble sleeping due to various environmental factors of the UTI. In this sense, given the care reality in an ICU, the question is: what care interventions are performed by the health team and what is their influence on the sleep continuity of the patients?

While it is recognized that sleep is important for the recovery of the health of the individual, no Brazilian studies were found that investigated the association between the healthcare interventions and the sleep of ICU patients. It is believed that the identification of the care interventions that interrupt the sleep of ICU patients can contribute to better planning of care and preserve the sleep patterns of patients.

Therefore, this study aimed to identify the care interventions performed by the health team and their influence on the sleep continuity of patients hospitalized in an ICU.

\section{Method}

This was a descriptive study, carried out in an adult ICU of a public hospital in Belo Horizonte, Brazil. The ICU has a capacity of 30 beds, and a care team composed of nurses, nursing technicians, physicians, physiotherapists and psychologists.

Data were collected from June to November 2011. During this period, 900 adult patients were admitted to the ICU. However, the study only included the patients that met the following inclusion criteria: aged over 18 years, length of stay in ICU greater than 24 hours, score of 15 on the Glasgow Coma Scale (GCS) and presentation of the nursing diagnosis "disturbed sleep pattern". The GCS score was justified by the need for interaction with the patient to evaluate the presence of the nursing diagnosis "disturbed sleep pattern", which was evaluated using an instrument developed by the researchers containing related factors and defining characteristics described in the NANDA-I(12) taxonomy, 
and the demographic data and clinical characteristics of the patients.

Patients were excluded from the study when: there was a diagnosis of neurological and/or mental disease and/or dementia, reports of sleep problems starting prior to admission, use of sedatives, opioids in high doses, muscle blockers, tricyclic antidepressants and/ or benzodiazepines within the preceding 24 hours, hemodynamic instability characterized by systolic blood pressure less than $90 \mathrm{mmHg}$, and presentation of mental confusion and/or psychomotor agitation. These criteria were included because they are considered confounding variables for the outcome evaluated, since, when present, they can prejudice the emission of information by the patients and/or change the measurement of the awakenings.

The sample was calculated from a pilot study with five patients. The calculation was based on the margin of error of the mean of the more frequent care interventions and on the total number of care interventions. The sample size was calculated considering the rule that: var $(\bar{X}=\operatorname{var}(x) / n$, where "var"=variance, " $x$ " is the frequency of the determined intervention, " $\bar{X}$ " is the mean of this frequency and " $n$ " is the sample size. It was found that, for a sample of up to 11 patients, the margin of error of the mean of the care interventions was greater than two. However, with a sample size equal to or greater than 12 patients, this margin of error tended to fall to values below two. Thus, a sample of 12 patients was established for the performance of the study.

To identify the care interventions carried out by the care team, a filming technique was used with a SONY brand, DCR-SX63 model, camcorder, which was placed on a pedestal and positioned in front of the cubical of each patient. At the end of the filming, the data were transferred to a computer and the images reproduced using the Picture Motion Browser software.

For the recording of the interventions, an instrument was used that was developed from a literature review and the clinical experience of the researchers. This was tested in the pilot study. The instrument consisted of 34 different care interventions, grouped into the blocks: feeding, hygiene and comfort care, tube, probe and drain care, respiratory care, performance of examinations and invasive procedures, evaluation of health status, and drug and non-drug therapy. For each intervention, the time of its occurrence was recorded.

The Actisleep actigraph equipment was used to evaluate the influence of the care interventions on the sleep continuity of the patients. This device allows the measurement of sleep disruption by detecting the awakening of the patient. The apparatus was placed on the wrist of the patients and surrounded by transparent film, changed every 24 hour period in each patient. It was not always possible to fix the device to the dominant limb, due to the presence on the wrist of monitoring and therapeutic devices, such as peripheral venous access and intra-arterial pressure catheter. The data registered by the actigraph was transferred to the computer and the analysis was carried out using the Actilife, version 5 , software. The registration performed by the actigraph allowed the awakening of the individuals according to the care interventions to be estimated. Awakenings arising from the care interventions were defined as those between 1 minute before and one minute after the end of the intervention.

For the processing and analysis of the data, the Statistical Package for the Social Sciences (SPSS), version 14.0, was used. Descriptive analysis was used for the characterization of the patients regarding gender and age, identifying the frequency of the care interventions and the interruptions in the sleep of the patients, using frequency distribution tables, measures of central tendency (mean and median) and variability measures (standard deviation).

The study was approved by the Research Ethics Committee (COEP) of the Federal University of Minas Gerais, COEP/UFMG process, authorization No. ETIC 0544.0.203.000-10.

\section{Results}

Of the 12 patients studied, nine (75\%) were male and three (25\%) female. Their ages ranged between 42 and 73 years, with a mean of 58 and standard deviation of 10.5 years. The most frequent medical diagnoses were: heart disease (4-33.4\%) shock/sepsis (3-25\%), followed by gastrointestinal disease, renal disease, metabolic disorders and pulmonary disease. All the patients were using at least one type of invasive device. The length of hospitalization ranged between 3 and 106 days, with a mean of 16.6 days and standard deviation of 29.4 days.

The defining characteristics of the "disturbed sleep pattern" Nursing Diagnosis (ND) identified most frequently (5-41.6\%) in the patients were "report of staying awake" and "report of difficulty sleeping". Of the 13 related factors described in the NANDA- $\mathrm{I}^{(12)}$ for the "disturbed sleep pattern" ND, four (30.7\%) were identified in the patients: "interruptions", "noise", "unfamiliar sleep furnishings" and "ambient temperature". 
During the data collection period, the health team conducted 28 different types of care interventions with the study patients, totaling 529 interventions, approximately 44.1 care interventions/patient every 24 hours, which represents a mean of 1.8 interventions/hour for each patient. Among the 28 types of interventions identified, six (21.4\%) were performed with all the patients: measurement of vital signs (109-20.6\%), assessment of the patients (90-17.0\%), medication administration (84-15.9\%), measurement of blood glucose (41-7.8\%), blood collection (from $17-7.4 \%$ ) and bathing (12-2.3\%), as shown in Table 1.

The 529 care interventions were performed with the patients throughout the 24 hours period, as shown in Figure 1.

The total filming time of this study was 288 hours. Of these, care interventions were not observed in only 63 hours $(21.8 \%)$, as shown in Figure 2.

Of the 529 care interventions, 21 (4.0\%) caused disruptions in the sleep of the patients. Of the 28 different types of care interventions performed, 12 $(42.8 \%)$ caused disruptions in the sleep of the patients. A total of 21 awakenings were registered. Of these, 13 (62\%) occurred during the daytime period, between $7.00 \mathrm{am}$ and $1.00 \mathrm{pm}$, and eight (38.0\%) at night, between midnight and 3.00am.
Table 1 - Care interventions conducted more frequently by the health team with the patients included in the study. Belo Horizonte, MG, Brazil, 2011

\begin{tabular}{|c|c|c|}
\hline Intervention & $\mathrm{n}^{*}$ & $\%$ \\
\hline Measurement of vital signs & 109 & 20.6 \\
\hline Patient assessment & 90 & 17.0 \\
\hline Drug administration & 84 & 15.9 \\
\hline Oral feeding administration & 72 & 13.6 \\
\hline Capillary blood glucose measurement & 41 & 7.8 \\
\hline Position change & 18 & 3.4 \\
\hline Blood collection & 17 & 3.2 \\
\hline Dressing change & 14 & 2.6 \\
\hline Change of bed linen and/or diapers & 14 & 2.6 \\
\hline Bath & 12 & 2.3 \\
\hline Micronebulization & 8 & 1.5 \\
\hline Oral cavity care & 7 & 1.3 \\
\hline Peripheral venous access maintenance & 7 & 1.3 \\
\hline Removal of invasive devices & 6 & 1.1 \\
\hline Hemodialysis & 5 & 0.9 \\
\hline X-ray in the bed & 4 & 0.8 \\
\hline Airway aspiration & 3 & 0.6 \\
\hline Peripheral venous access puncture & 3 & 0.6 \\
\hline Electrocardiogram & 3 & 0.6 \\
\hline Invasive procedures & 2 & 0.4 \\
\hline Gastric/enteric intubation & 2 & 0.4 \\
\hline Swab collection & 2 & 0.4 \\
\hline Blood product administration & 1 & 0.2 \\
\hline Feeding by enteric tube & 1 & 0.2 \\
\hline Gastric stasis measurement & 1 & 0.2 \\
\hline Noninvasive ventilation & 1 & 0.2 \\
\hline Tracheostomy Care & 1 & 0.2 \\
\hline Echocardiogram & 1 & 0.2 \\
\hline Total & 529 & 100 \\
\hline
\end{tabular}

*Number of times the intervention was implemented.

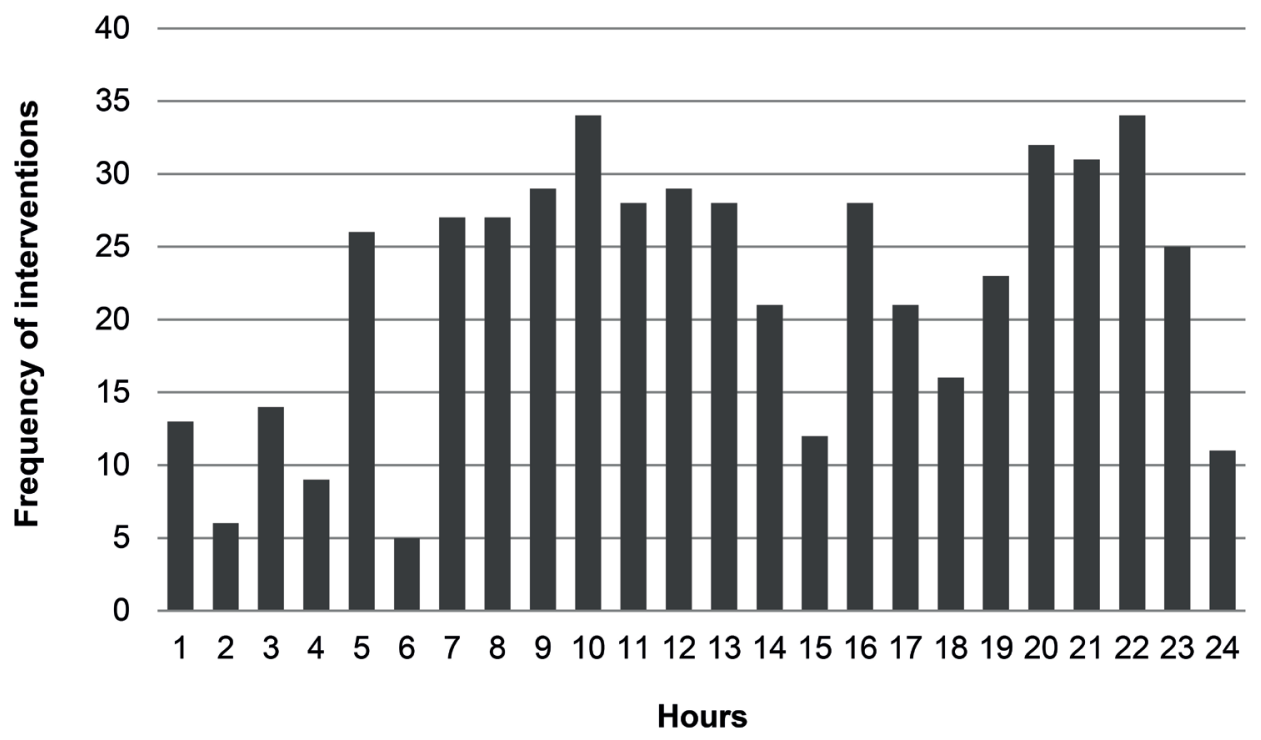

Figure 1 - Temporal distribution of the care interventions provided to the patients included in the study. Belo Horizonte, MG, Brazil, 2011 


\begin{tabular}{|c|c|c|c|c|c|c|c|c|c|c|c|c|c|c|c|c|c|c|c|c|c|c|c|c|}
\hline Patient & 7 & 8 & 9 & 10 & 11 & 12 & 13 & 14 & 15 & 16 & 17 & 18 & 19 & 20 & 21 & 22 & 23 & 24 & 1 & 2 & 3 & 4 & 5 & 6 \\
\hline 1 & & & & & & & & & & & & & & & & & & & & & & & & \\
\hline 2 & & & & & & & & & & & & & & & & & & & & & & & & \\
\hline 3 & & & & & & & & & & & & & & & & & & & & & & & & \\
\hline 4 & & & & & & & & & & & & & & & & & & & & 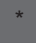 & & & & \\
\hline 5 & & & & & & & & & & & & & & & & & & * & & * & & & & \\
\hline 6 & & & & & & & & & & * & & & & & & * & & & * & & & * & & * \\
\hline 7 & & & & & & & & * & & & & & & & & & & & & & & 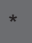 & & \\
\hline 8 & & & & & & & & & & & & & & & & & * & & & * & & & & * \\
\hline 9 & & & & & & & & & & & & & & * & & & & . & & * & & * & & \\
\hline 10 & & & & & & & & & & & & & & & & & & * & * & & & & & \\
\hline 11 & * & * & & & & * & & & & * & & & & * & & & & & & & * & * & & * \\
\hline 12 & & & & & & & & & & & & & & & & & & & & & * & & & \\
\hline
\end{tabular}

*Highlighted cells represent the hours in which care interventions were not carried out.

Figure 2 - Distribution of hours in which care interventions were not carried out per patient evaluated. Belo Horizonte, MG, Brazil, 2011

\section{Discussion}

The defining characteristics of the "disturbed sleep pattern" ND, identified with higher frequencies in the patients were: "report of staying awake" (5$41.6 \%$ ) and "report of difficulty sleeping" (5-41.6\%), a result that was also found in other studies in which ICU patients reported difficulty sleeping(3,11,14-15). The "disturbed sleep pattern" ND was identified among the five most frequent diagnoses in ICU patients and the defining characteristic "report of difficulty sleeping" was present in 23 (95.8\%) patients ${ }^{(16)}$. These findings highlight the magnitude of this nursing problem in ICU patients, which requires the planning of interventions to improve the sleep of patients.

Among the related factors of the "disturbed sleep pattern" ND described in the taxonomy of the NANDA-I ${ }^{(12)}$, four (30.7\%) were identified in the patients of this study. Noise was reported by three patients (25\%) as a factor that disturbed their sleep. A study of noise measurement in the ICU found high noise levels, both during the day and at night ${ }^{(17)}$. Noise has been identified as the main environmental factor causing changes in the sleep pattern ${ }^{(18)}$. It is therefore recommended that, among the nursing interventions aimed at improving the sleep of ICU patients, those directed toward reducing environmental noise are included, since this is one of the main disturbances of sleep.

Interruptions for therapeutic reasons, monitoring and laboratory tests are also causes of awakening from sleep ${ }^{(12)}$. In this study, this related factor was identified in three $(25 \%)$ of the patients with the "disturbed sleep pattern" ND(12), confirming similar findings(19-22). As well as noise control, the planning of the nursing care for the performance of these interventions should, where possible, take into consideration the time needed to ensure quality sleep for the patients, since this factor interferes with the recovery of health.

Although not described in the NANDA-I ${ }^{(12)}$, pain and the presence of tubes and probes were highlighted by the patients as factors related to sleep disruption in the ICU. A similar study(16) found that pain was reported by $59.1 \%$ of the patients as a factor that disturbed their sleep. The NANDA-I ${ }^{(12)}$ describes "disturbed sleep" as a defining characteristic of the "acute pain" ND. However, pain is not described as a related factor for the "disturbed sleep pattern" ND(12). To date, few studies have investigated the relationship between pain and sleep quality in the intensive care environment. It is known that it interferes with sleep, however, lack of sleep can increase the perception of pain ${ }^{(23)}$. With regard to the presence of tubes and probes as factors that disturb the sleep, in one study ${ }^{(24)}$ that aimed to identify the physical and psychological stressors of ICU hospitalization, the presence of tubes in the mouth or nose was highlighted as the main stress factor.

Given the above, further studies are recommend aimed at validating the related factors and defining characteristics of the "disturbed sleep pattern" ND, especially those highlighted in this study and that are not described in the taxonomy of the NANDA-I ${ }^{(12)}$, thereby contributing to the improvement of this classification.

In this study, 28 different types of care interventions were identified, with a mean of 44.1 care interventions 
in a 24 hour period per patient. This finding, although it reflects a high number of care interventions, is less than that of other studies(22,25), which identified, respectively, means of 42.6 and 51 care interventions/patient during the night period. The lower mean number of interventions performed with the patients of this study can be explained by the fact that they were patients with lower severity levels than the majority of ICU patients, justified by the inclusion criteria of the study. However, even dealing with less severe patients, there was a mean of 1.8 care interventions/hour for each patient. In a similar study(21), the performance of at least one intervention/hour for each patient was observed over the 24 hours of the day. Both findings demonstrate how patients have little opportunity to sleep in the intensive care environment.

The care interventions more frequently performed with the patients were: measurement of vital signs, patient assessment, drug administration, oral feeding administration and measurement of blood glucose. In another study ${ }^{(22)}$ the interventions identified as the most frequent in an ICU were measurement of vital signs, drug administration, patient assessments, changes of position and manipulation of the mechanical ventilator, all of which are the responsibility of the nursing team. Therefore, it is believed that better planning of these interventions, according to the demand of each patient, can help to reduce the frequency of interruptions and consequently improve the sleep of ICU patients.

A high frequency of care interventions at night was observed (229-43.3\%), with occurrence peaks between $8.00 \mathrm{pm}$ and $10.00 \mathrm{pm}$ and at 5.00am, which is in agreement with similar findings ${ }^{(22,25)}$ observed in other studies, which showed the highest frequency of interventions at $8.00 \mathrm{pm}$, midnight and 6.00am, and at 8.00pm, midnight and between 2.00am and 5.00am, respectively. In the three studies $8.00 \mathrm{pm}$ was found to be one of the peak times for the performance of care interventions. This finding coincides with the start of the night shift in the institutions under study, which may explain the peak of interventions at that time.

It was found that the performance of care interventions with the patients was not observed in only $21 \%$ (63 hours) of the hours of filming. Of the 12 patients studied, only one presented four uninterrupted nighttime hours without interventions being conducting and three presented nighttime periods of 120 uninterrupted minutes without any intervention (Figure 2 ). This finding is corroborated by a study(21) that also did not find any period longer than four consecutive hours without the performance of interventions with the patients. Thus, it can be inferred that, even if the patients had ideal conditions for sleep, the performance of care interventions would not allow a complete sleep cycle of approximately 90 minutes to take place, the restorative time required for an individual.

Of the 529 care interventions performed, 21 (4.0\%) caused disruptions in the sleep of the patients. Of the 28 different types of care interventions performed, 12 $(42.8 \%)$ caused disruptions in the sleep of the patients. Although the number of awakenings appeared low, in one study ${ }^{(20)}$, designed to determine the impact of noise and care interventions on the sleep continuity of patients, it was found that $17.7 \%$ of the care interventions resulted in disturbances in the sleep pattern and were responsible for $7 \%$ of all the awakenings measured. It should also be noted that actigraphy has limitations for the evaluation of sleep, as previously described. Given the above, further studies are recommended using more accurate methods to evaluate the sleep of patients and its relationship with the interventions conducted by the health team, especially the nursing staff.

\section{Conclusion}

Based on the awakenings from sleep of the patients studied, it was concluded that care interventions can impair the sleep of ICU patients. It was perceived that the interventions that caused awakenings were performed at times that suited the routine of the ICU professionals, at the expense of the preferences or needs of the individuals.

It is estimated that the lack of concern of the professionals with the quality of sleep in ICU patients is related to the lack of discussion of the topic in the clinical practice. Thus, it is recommended that quality of sleep is included in the discussions agenda of the professional, so that sleep is also a focus of the nursing care in ICUs.

The use of the actigraph to measure sleep disruptions was a limiting factor of the study. This apparatus is not capable of measuring the sleep architecture of patients and its sensitivity to identify sleep awakenings is low. It was observed that the device was not able to distinguish the times when the patient was awake and immobile from those in which the patient was sleeping, nor could it distinguish whether the movements of the patient occurred during periods of sleep or when awake. Some awakening registrations could have been false due to movement of the patient while asleep. It was possible to circumvent these limitations by comparing the actigraph 
data with the images obtained by the filming. Therefore, the choice of the device for the measurement of sleep awakenings was considered adequate.

The results of this study are fundamental to perceive the fragility of sleep for ICU patients. It is known that sleep quality is a constant challenge for patients and health professionals. Changes in the sleep pattern of these individuals is a multifactorial disorder and difficult to control. Given the above, the need to standardize care actions can be perceived, differentiating them according to the characteristics of the patients and the level of severity of their health, to provide uninterrupted periods of sleep, encouraging higher quality care and contributing to the more rapid and satisfactory recovery of hospitalized individuals.

\section{References}

1. Ministério da Saúde (BR). Portaria MS 3432, 12 de Agosto de 1998. Dispõe sobre critérios de classificação entre unidades de tratamento intensivo [Internet]. Brasília; 1998. [acesso 1 nov 2011]. Disponível em: dtr2001.saude.gov.br/sas/PORTARIAS/PORT98/GM/ PRT-3432.pdf.

2. Elliott RA, Mckinley SA, Cistulli P. The quality and duration of sleep in the intensive care setting: an integrative review. Int J Nurs Stud. 2011;48:384-400.

3. Ehlers VJ, Watson $\mathrm{H}$, Moleki MM. Factors contributing to sleep deprivation in a multi-disciplinary intensive care unit in South Africa. Curationis. 2013;36(1):1-8.

4. Waye KP, Elmenhorst EM, Croy I, Pedersen E. Improvement of intensive care unit sound environment and analyses of consequences on sleep: an experimental study. Sleep Med. 2013;14(12):1334-40.

5. Makic MBF, Rauen C, Watson R, Poteet AWL. Examining the evidence to guide practice: challenging practice habits. Crit Care Nurs. 2014;34:28-45.

6. Pulak LM, Jensen L. Sleep in the intensive care unit: a review. J Intensive Care Med [Internet] 2014. [acesso 30 ago 2014]. Disponível em: http://jic.sagepub.com/ content/early/2014/06/03/0885066614538749.full

7. Guimarães GM. Diagnóstico polissonográfico. Pulmão. (Rio de Janeiro) 2010;9(3-4):88-92.

8. Kamdar BB, Needham DM, Collop AA. Sleep deprivation in critical ilness: it's role in physical and psychological recovery. J Intensive Care Med. 2012;27(2):97-111.

9. Monteiro ES. Revisão aberta do artigo "Significado Clínico da Actigrafia". Rev Neurociênc. 2011;19(1):162-4. 10. Togeiro SMGP, Smith AK. Métodos diagnósticos nos distúrbios do sono. Rev Bras Psiquiatr. 2005;27:8-15.
11. Elliott R, McKinley S, Cistulli P, Fien M. Characterisation of sleep in intensive care using 24-hour polysomnography: an observational study. Crit Care. [Internet] 2013. [acesso 30 ago 2014]. Disponível em: http://www. biomedcentral.com/content/pdf/cc12565.pdf

12. North American Nursing Diagnosis AssociationNANDA International. Diagnósticos de Enfermagem da NANDA: definições e classificação 2012-2014. Porto Alegre: Artmed; 2013. 606 p.

13. Magalhães MN, Lima A. Noções de probabilidade e estatística. 7ed. São Paulo: IME-USP; 2007. p. 416.

14. Frisk $U$, Nordstro MG. Patients sleep in an intensive care unit patients' and nurses' perception. Intensive Crit Care Nurs. 2003;19:342-9.

15. Bihari S, McEvoy RD, Matheson E, Kim S, Woodman RJ. Factors affecting sleep quality of patients in intensive care unit. J Clin Sleep Med. 2012;8(3):301-7.

16. Pasini D, Alvim I, Kanda L, Mendes RSP, Cruz DALM. Diagnósticos de enfermagem de pacientes internados em unidades de terapia intensiva. Rev Esc Enferm USP. 1996;30(3):501-18.

17. Lawson N, Thompson K, Saunders G, Saiz J, Richardson J, Brown D, et al. Sound intensity and noise evaluation in a critical care unit. Am J Respir Crit Care Med. 2010;19:88-99.

18. Ugras GA, Öztekin SD. Patient perception of environmental and nursing factors contributing to sleep disturbances in a neurosurgical intensive care unit. Tohoku J Exp Med. 2007;212(3):299-308.

19. Freedman NS, Kotzer N, Schwab RJ. Patient perception of sleep quality and etiology of sleep disruption in the intensive care unit. Am J Respir Crit Care Med. 1999;159:1155-62.

20. Gabor JY, Cooper AB, Crombach SA, Lee B, Kadikar $\mathrm{N}$, Bettger $\mathrm{HE}$, et al. Contribution of the intensive care unit environment to sleep disruption in mechanically ventilated patients and healthy subjects. Am J Respir Crit Care Med. 2003;167:708-15.

21. Meyer TJ, Eveloff SE, Bauer MS, Schwartz WA, Hill NS, Millman RP. Adverse environmental conditions in the respiratory and medical ICU settings. Chest. 1994; 105; 1211-6.

22. Tamburri LM, Dibrienza R, Zozula R, Redeker NS. Nocturnal care interactions with patients in critical care units. Am J Crit Care. 2004;13(2):102-22.

23. Redeker NS. Sleep in acute care settings: a integrative review. J Nurs Scholarsh. 2000;32(1):31-8.

24. Hweidi IM. Jordanian patients' perception of stressors in critical care units: a questionnaire survey. Int J Nurs Stud. 2007;44:227-35. 
25. Çelik S, Oztekin D, Akyolcu N, Issever H. Sleep disturbance: the patient care activities applied at the night shift in the intensive care unit. J Clin Nurs.

2005; 14:102-6. 